\title{
A new generalization of some quantum integral inequalities for quantum differentiable convex functions
}

\author{
Yi-Xia Li', Muhammad Aamir Ali², Hüseyin Budak ${ }^{3}$, Mujahid Abbas ${ }^{4}$ and Yu-Ming Chu ${ }^{5^{*}}$ (D)
}

\section{"Correspondence:}

chuyuming2005@126.com

${ }^{5}$ Department of Mathematics,

Huzhou University, Huzhou 313000,

China

Full list of author information is

available at the end of the article

\section{Springer}

\begin{abstract}
In this paper, we offer a new quantum integral identity, the result is then used to obtain some new estimates of Hermite-Hadamard inequalities for quantum integrals. The results presented in this paper are generalizations of the comparable results in the literature on Hermite-Hadamard inequalities. Several inequalities, such as the midpoint-like integral inequality, the Simpson-like integral inequality, the averaged midpoint-trapezoid-like integral inequality, and the trapezoid-like integral inequality, are obtained as special cases of our main results.
\end{abstract}

MSC: 26D10; 26D15; 26A51

Keywords: Hermite-Hadamard inequality; Trapezoid inequalities; Midpoint inequalities; Quantum calculus; Convex functions

\section{Introduction}

The study of $q$-calculus was initiated in the early 20th century after the work of Jackson (1910) who defined an integral later known as the $q$-Jackson integral (see [16, 22, 23, 27, 28]). In $q$-calculus, the classical derivative is replaced by the $q$-difference operator to deal with nondifferentiable functions. For more discussion on this subject, we refer to [8, 21]. Applications of $q$-calculus can be found in various disciplines of mathematics and physics (see [13, 26, 36, 45]).

Many well-known integral inequalities, such as Hölder inequality, Hermite-Hadamard inequalities and Ostrowski inequality, Cauchy-Bunyakovsky-Schwarz inequality, Grüss inequality, Grüss-Chebysev inequality, and other integral inequalities, have been studied in the setup of $q$-calculus using the concept of classical convexity. For more results in this direction, we refer to $[1-7,10,11,14,18-20,24,29-32,34,35,37,39-43,46,47]$.

The purpose of this paper is to prove several new quantum integral inequalities by applying the newly defined concept of a $q^{b}$-integral. We also discuss the relation of our results with comparable results existing in the literature.

The organization of this paper is as follows: In Sect. 2, a brief description of the concepts of $q$-calculus and some related works in this direction are given. In Sect. 3, the bounds of Hermite-Hadamard-type inequalities for the $q^{b}$-integrals are presented. In Sect. 4, several

(c) The Author(s) 2021. This article is licensed under a Creative Commons Attribution 4.0 International License, which permits use, sharing, adaptation, distribution and reproduction in any medium or format, as long as you give appropriate credit to the original author(s) and the source, provide a link to the Creative Commons licence, and indicate if changes were made. The images or other third party material in this article are included in the article's Creative Commons licence, unless indicated otherwise in a credit line to the material. If material is not included in the article's Creative Commons licence and your intended use is not permitted by statutory regulation or exceeds the permitted use, you will need to obtain permission directly from the copyright holder. To view a copy of this licence, visit http://creativecommons.org/licenses/by/4.0/. 
special cases of our main results are discussed. The relationship between the results presented herein and comparable results in the literature is also studied. Section 5 contains some conclusions and further directions for future research. We believe that the study initiated in this paper may inspire new research in this area.

\section{Preliminaries of $q$-calculus and some inequalities}

In this section, we first present some known definitions and related inequalities in the $q$-calculus. Set the following notation (see [28]):

$$
[n]_{q}=\frac{1-q^{n}}{1-q}=1+q+q^{2}+\cdots+q^{n-1}, \quad q \in(0,1) .
$$

Jackson [27] defined the $q$-Jackson integral of a given function $f$ from 0 to $b$ as follows:

$$
\int_{0}^{b} f(x) d_{q} x=(1-q) b \sum_{n=0}^{\infty} q^{n} f\left(b q^{n}\right), \quad \text { where } 0<q<1,
$$

provided that the sum converges absolutely.

Jackson [27] defined the $q$-Jackson integral of a given function over the interval $[a, b]$ as follows:

$$
\int_{a}^{b} f(x) d_{q} x=\int_{0}^{b} f(x) d_{q} x-\int_{0}^{a} f(x) d_{q} x .
$$

Definition 1 ([38]) Let $f:[a, b] \rightarrow \mathbb{R}$ be a function. The $q_{a}$-derivative of $f$ at $x \in[a, b]$ is identified by the the following expression:

$$
{ }_{a} D_{q} f(x)=\frac{f(x)-f(q x+(1-q) a)}{(1-q)(x-a)}, \quad x \neq a .
$$

If $x=a$, we define ${ }_{a} D_{q} f(a)=\lim _{x \rightarrow a} D_{q} f(x)$ if it exists and is finite.

Definition 2 ([15]) Let $f:[a, b] \rightarrow \mathbb{R}$ be a function. The $q^{b}$-derivative of $f$ at $x \in[a, b]$ is given by

$$
{ }^{b} D_{q} f(x)=\frac{f(q x+(1-q) b)-f(x)}{(1-q)(b-x)}, \quad x \neq b .
$$

If $x=b$, we define ${ }^{b} D_{q} f(b)=\lim _{x \rightarrow b}{ }^{b} D_{q} f(x)$ if it exists and is finite.

Definition 3 ([38]) Let $f:[a, b] \rightarrow \mathbb{R}$ be a function. Then, the $q_{a}$-definite integral on $[a, b]$ is defined by

$$
\begin{aligned}
\int_{a}^{b} f(x)_{a} d_{q} x & =(1-q)(b-a) \sum_{n=0}^{\infty} q^{n} f\left(q^{n} b+\left(1-q^{n}\right) a\right) \\
& =(b-a) \int_{0}^{1} f((1-t) a+t b) d_{q} t .
\end{aligned}
$$

Alp et al. [11] proved the following $q_{a}$-Hermite-Hadamard inequalities for convex functions in the setting of quantum calculus: 
Theorem 1 Iff $:[a, b] \rightarrow \mathbb{R}$ is a convex differentiable function on $[a, b]$ and $0<q<1$. Then we have

$$
f\left(\frac{q a+b}{[2]_{q}}\right) \leq \frac{1}{b-a} \int_{a}^{b} f(x)_{a} d_{q} x \leq \frac{q f(a)+f(b)}{[2]_{q}} .
$$

In [11] and [33], the authors established some bounds for the left- and right-hand sides of the inequality (2.3).

On the other hand, Bermudo et al. [15] gave the following definition and obtained the related Hermite-Hadamard-type inequalities:

Definition 4 ([15]) Let $f:[a, b] \rightarrow \mathbb{R}$ be a function. Then, the $q^{b}$-definite integral on $[a, b]$ is given by

$$
\begin{aligned}
\int_{a}^{b} f(x)^{b} d_{q} x & =(1-q)(b-a) \sum_{n=0}^{\infty} q^{n} f\left(q^{n} a+\left(1-q^{n}\right) b\right) \\
& =(b-a) \int_{0}^{1} f(t a+(1-t) b) d_{q} t .
\end{aligned}
$$

Theorem 2 ([15]) Iff $:[a, b] \rightarrow \mathbb{R}$ is a convex, differentiable function on $[a, b]$ and $0<q<$ 1, then the q-Hermite-Hadamard inequalities are given as follows:

$$
f\left(\frac{a+q b}{[2]_{q}}\right) \leq \frac{1}{b-a} \int_{a}^{b} f(x)^{b} d_{q} x \leq \frac{f(a)+q f(b)}{[2]_{q}} .
$$

From Theorems 1 and 2, one can obtain the following inequalities:

Corollary 1 ([15]) For any convex function $f:[a, b] \rightarrow \mathbb{R}$ and $0<q<1$, we have

$$
\begin{aligned}
f\left(\frac{q a+b}{[2]_{q}}\right)+f\left(\frac{a+q b}{[2]_{q}}\right) & \leq \frac{1}{b-a}\left\{\int_{a}^{b} f(x)_{a} d_{q} x+\int_{a}^{b} f(x)^{b} d_{q} x\right\} \\
& \leq f(a)+f(b)
\end{aligned}
$$

and

$$
f\left(\frac{a+b}{2}\right) \leq \frac{1}{2(b-a)}\left\{\int_{a}^{b} f(x)_{a} d_{q} x+\int_{a}^{b} f(x)^{b} d_{q} x\right\} \leq \frac{f(a)+f(b)}{2} .
$$

We recall the following well-known inequality:

Theorem 3 (Hölder's inequality, [12, p. 604]) Let $x>0,0<q<1, p_{1}>1$ be such that $\frac{1}{p_{1}}+$ $\frac{1}{r_{1}}=1$. Then

$$
\int_{0}^{x}|f(x) g(x)| d_{q} x \leq\left(\int_{0}^{x}|f(x)|^{p_{1}} d_{q} x\right)^{\frac{1}{p_{1}}}\left(\int_{0}^{x}|g(x)|^{r_{1}} d_{q} x\right)^{\frac{1}{r_{1}}} .
$$




\section{Main results}

In this section, we give some new estimates of Hermite-Hadamard-type inequalities for functions whose first $q^{b}$-derivatives in absolute value are convex.

Let's start with the following useful lemma.

Lemma 1 If $:[a, b] \subset \mathbb{R} \rightarrow \mathbb{R}$ is a $q^{b}$-differentiable function on $(a, b)$ such that ${ }^{b} D_{q} f$ is continuous and integrable on $[a, b]$, then we have

$$
\begin{aligned}
(b-a) & {\left[\int_{0}^{v}(q t+\gamma v-\gamma)^{b} D_{q} f(t a+(1-t) b) d_{q} t\right.} \\
& \left.+\int_{v}^{1}(q t+\gamma v-1)^{b} D_{q} f(t a+(1-t) b) d_{q} t\right] \\
= & \frac{1}{b-a} \int_{a}^{b} f(x)^{b} d_{q} x-\gamma[v f(a)+(1-v) f(b)]-(1-\gamma) f(v a+(1-v) b),
\end{aligned}
$$

where $0<q<1$.

Proof From Definition 2, it follows that

$$
{ }^{b} D_{q} f(t a+(1-t) b)=\frac{f(q t a+(1-q t) b)-f(t a+(1-t) b)}{(1-q)(b-a) t} .
$$

By applying identical transformation, we obtain

$$
\begin{aligned}
& (b-a) \int_{0}^{v}(q t+\gamma v-\gamma)^{b} D_{q} f(t a+(1-t) b) d_{q} t \\
& \quad+(b-a) \int_{v}^{1}(q t+\gamma v-1)^{b} D_{q} f(t a+(1-t) b) d_{q} t \\
& =(b-a) \int_{0}^{1}(q t+\gamma v-1)^{b} D_{q} f(t a+(1-t) b) d_{q} t \\
& \quad+(b-a) \int_{0}^{v}(1-\gamma)^{b} D_{q} f(t a+(1-t) b) d_{q} t .
\end{aligned}
$$

By the equality (2.1), we obtain

$$
\begin{aligned}
& \int_{0}^{v}{ }^{b} D_{q} f(t a+(1-t) b) d_{q} t \\
& \quad=\int_{0}^{v} \frac{f(q t a+(1-q t) b)-f(t a+(1-t) b)}{(1-q)(b-a) t} d_{q} t \\
& \quad=\frac{1}{b-a}\left[\sum_{n=0}^{\infty} f\left(q^{n+1} v a+\left(1-q^{n+1} v\right) b\right)-\sum_{n=0}^{\infty} f\left(q^{n} v a+\left(1-q^{n} v\right) b\right)\right] \\
& \quad=\frac{1}{b-a}[f(b)-f(v a+(1-v) b)]
\end{aligned}
$$


and

$$
\begin{aligned}
& \int_{0}^{1}{ }^{b} D_{q} f(t a+(1-t) b) d_{q} t \\
& \quad=\int_{0}^{1} \frac{f(q t a+(1-q t) b)-f(t a+(1-t) b)}{(1-q)(b-a) t} d_{q} t \\
& \quad=\frac{1}{b-a}\left[\sum_{n=0}^{\infty} f\left(q^{n+1} a+\left(1-q^{n+1}\right) b\right)-\sum_{n=0}^{\infty} f\left(q^{n} a+\left(1-q^{n}\right) b\right)\right] \\
& \quad=\frac{1}{b-a}[f(b)-f(a)] .
\end{aligned}
$$

It follows from (2.1) and Definition 4 that

$$
\begin{aligned}
\int_{0}^{1} t^{b} D_{q} f(t a+(1-t) b) d_{q} t & \\
= & \int_{0}^{1} \frac{f(q t a+(1-q t) b)-f(t a+(1-t) b)}{(1-q)(b-a)} d_{q} t \\
= & \frac{1}{b-a}\left[\sum_{n=0}^{\infty} q^{n} f\left(q^{n+1} a+\left(1-q^{n+1}\right) b\right)-\sum_{n=0}^{\infty} q^{n} f\left(q^{n} a+\left(1-q^{n}\right) b\right)\right] \\
= & \frac{1}{b-a}\left[\frac{1}{q} \sum_{n=0}^{\infty} q^{n+1} f\left(q^{n+1} a+\left(1-q^{n+1}\right) b\right)-\sum_{n=0}^{\infty} q^{n} f\left(q^{n} a+\left(1-q^{n}\right) b\right)\right] \\
= & \frac{1}{b-a}\left[\frac{1}{q} \sum_{n=0}^{\infty} q^{n} f\left(q^{n} a+\left(1-q^{n}\right) b\right)-\frac{1}{q} f(a)-\sum_{n=0}^{\infty} q^{n} f\left(q^{n} a+\left(1-q^{n}\right) b\right)\right] \\
= & \frac{1}{b-a}\left[\left(\frac{1}{q}-1\right) \sum_{n=0}^{\infty} q^{n} f\left(q^{n} a+\left(1-q^{n}\right) b\right)-\frac{1}{q} f(a)\right] \\
= & \frac{1}{b-a}\left(\frac{1-q}{q}\right) \sum_{n=0}^{\infty} q^{n} f\left(q^{n} a+\left(1-q^{n}\right) b\right)-\frac{1}{q(b-a)} f(a) \\
= & \left.\frac{1}{q(b-a)^{2}} \int_{a}^{b} f(x)^{b} d_{q^{x} x-\frac{1}{q(b-a)} f(a) .}\right]
\end{aligned}
$$

Using (3.3), (3.4), and (3.5) in (3.2), we obtain the required identity.

Remark 1 If we take $q \rightarrow 1^{-}$in the Lemma 1, then we have [25, Lemma 2.1].

Before we present our main inequalities, we first give some calculated quantum integrals:

$$
\begin{aligned}
A_{1 q}(\gamma, v) & =\int_{0}^{v} t|q t+\gamma v-\gamma| d_{q} t= \begin{cases}\frac{v^{3} q}{[3]_{q}}-\frac{v^{2} \gamma(1-v)}{[2]_{q}}+\frac{2 \gamma^{3}(1-v)^{3}}{\left([2]_{q}\right)\left([3]_{q}\right)}, & (\gamma+q) v>\gamma, \\
\frac{v^{2} \gamma(1-v)}{[2]_{q}}-\frac{v^{3} q}{[3]_{q}}, & (\gamma+q) v \leq \gamma,\end{cases} \\
B_{1 q}(\gamma, v) & =\int_{0}^{v}(1-t)|q t+\gamma v-\gamma| d_{q} t \\
& =\int_{0}^{v}|q t+\gamma v-\gamma| d_{q} t-\int_{0}^{v} t|q t+\gamma v-\gamma| d_{q} t
\end{aligned}
$$




$$
\begin{aligned}
& = \begin{cases}\frac{v^{2} q+2 \gamma^{2}(1-v)^{2}+v^{2} \gamma(1-v)}{[2]_{q}}-v \gamma(1-v)-\frac{v^{3} q}{[3]_{q}}-\frac{2 \gamma^{3}(1-v)^{3}}{\left([2]_{q}\right)\left([3]_{q}\right)}, & (\gamma+q) \nu>\gamma, \\
v \gamma(1-v)-\frac{v^{2} q+v^{2} \gamma(1-v)}{[2]_{q}}+\frac{\nu^{3} q}{[3]_{q}}, & (\gamma+q) v \leq \gamma,\end{cases} \\
& A_{2 q}(\gamma, v)=\int_{0}^{1} t|q t+\gamma \nu-1| d_{q} t= \begin{cases}\frac{q}{[3]_{q}}-\frac{1-\gamma v}{[2]_{q}}+\frac{2(1-\gamma v)^{3}}{\left([2]_{q}\right)\left([3]_{q}\right)}, & \gamma v+q>1, \\
\frac{1-\gamma v}{[2]_{q}}-\frac{q}{[3]_{q}}, & \gamma v+q \leq 1,\end{cases} \\
& B_{2 q}(\gamma, v)=\int_{0}^{1}(1-t)|q t+\gamma v-1| d_{q} t \\
& =\int_{0}^{1}|q t+\gamma v-1| d_{q} t-\int_{0}^{1} t|q t+\gamma \nu-1| d_{q} t \\
& = \begin{cases}\frac{q+2(1-\gamma \nu)^{2}+1-\gamma \nu}{[2]_{q}}-(1-\gamma \nu)-\frac{q}{[3]_{q}}-\frac{2(1-\gamma \nu)^{3}}{\left([2]_{q}\right)\left([3]_{q}\right)}, & \gamma v+q>1, \\
1-\gamma \nu-\frac{q+1-\gamma v}{[2]_{q}}+\frac{q}{[3]_{q}}, & \gamma \nu+q \leq 1,\end{cases} \\
& A_{3 q}(\gamma, v)=\int_{0}^{v} t|q t+\gamma v-1| d_{q} t= \begin{cases}\frac{q v^{3}}{[3]_{q}}-\frac{v^{2}(1-\gamma v)}{[2]_{q}}+\frac{2(1-\gamma v)^{3}}{\left([2]_{q}\right)\left([3]_{q}\right)}, & \gamma v+q>1, \\
\frac{v^{2}(1-\gamma v)}{[2]_{q}}-\frac{q v^{3}}{[3]_{q}}, & \gamma v+q \leq 1,\end{cases} \\
& B_{3 q}(\gamma, v)=\int_{0}^{v}(1-t)|q t+\gamma v-1| d_{q} t \\
& =\int_{0}^{v}|q t+\gamma v-1| d_{q} t-\int_{0}^{v} t|q t+\gamma v-1| d_{q} t \\
& = \begin{cases}\frac{v^{2}+2(1-\gamma v)^{2}+v^{2}(1-\gamma v)}{[2]_{q}}-v(1-\gamma \nu)-\frac{q v^{3}}{[3]_{q}}-\frac{2(1-\gamma \nu)^{3}}{\left([2]_{q}\right)\left([3]_{q}\right)}, & \gamma v+q>1, \\
v(1-\gamma \nu)-\frac{q v^{2}+v^{2}(1-\gamma v)}{[2]_{q}}+\frac{q v^{3}}{[3]_{q}}, & \gamma v+q \leq 1,\end{cases} \\
& C_{1 q}(\gamma, v)=\int_{0}^{1}|q t+\gamma v-1| d_{q} t= \begin{cases}\frac{q+2(1-\gamma v)^{2}}{[2]_{q}}-(1-\gamma v), & \gamma v+q>1, \\
(1-\gamma v)-\frac{q}{[2]_{q}}, & \gamma v+q \leq 1 .\end{cases}
\end{aligned}
$$

Theorem 4 If $f:[a, b] \subset \mathbb{R} \rightarrow \mathbb{R}$ is a $q^{b}$-differentiable function on $(a, b)$ such that ${ }^{b} D_{q} f$ is continuous and integrable on $[a, b]$, then we have the following inequality provided that $\left.\right|^{b} D_{q} f \mid$ is convex on $[a, b]$ :

$$
\begin{aligned}
& \left|\frac{1}{b-a} \int_{a}^{b} f(x)^{b} d_{q} x-\gamma[v f(a)+(1-v) f(b)]-(1-\gamma) f(v a+(1-v) b)\right| \\
& \leq(b-a)\left[\left(A_{1 q}(\gamma, v)+A_{2 q}(\gamma, v)-A_{3 q}(\gamma, v)\right)\left|{ }^{b} D_{q} f(a)\right|\right. \\
& \left.\quad+\left.\left(B_{1 q}(\gamma, \nu)+B_{2 q}(\gamma, v)-B_{3 q}(\gamma, v)\right)\right|^{b} D_{q} f(b) \mid\right]
\end{aligned}
$$

where $0<q<1$.

Proof On taking the modulus in Lemma 1 and applying the convexity of $\left|{ }^{b} D_{q} f\right|$, we obtain

$$
\begin{aligned}
& \left|\frac{1}{b-a} \int_{a}^{b} f(x)^{b} d_{q} x-\gamma[v f(a)+(1-v) f(b)]-(1-\gamma) f(v a+(1-v) b)\right| \\
& \leq(b-a)\left[\left.\int_{0}^{v}|q t+\gamma v-\gamma|\right|^{b} D_{q} f(t a+(1-t) b) \mid d_{q} t\right. \\
& \left.\quad+\left.\int_{v}^{1}|q t+\gamma v-1|\right|^{b} D_{q} f(t a+(1-t) b) \mid d_{q} t\right]
\end{aligned}
$$




$$
\begin{aligned}
= & (b-a)\left[\left.\int_{0}^{v}|q t+\gamma v-\gamma|\right|^{b} D_{q} f(t a+(1-t) b) \mid d_{q} t\right. \\
& +\left.\int_{0}^{1}|q t+\gamma v-1|\right|^{b} D_{q} f(t a+(1-t) b) \mid d_{q} t \\
& \left.-\left.\int_{0}^{v}|q t+\gamma v-1|\right|^{b} D_{q} f(t a+(1-t) b) \mid d_{q} t\right] \\
\leq & (b-a)\left[\left|{ }^{b} D_{q} f(a)\right| \int_{0}^{v} t|q t+\gamma v-\gamma| d_{q} t+\left|{ }^{b} D_{q} f(b)\right| \int_{0}^{v}(1-t)|q t+\gamma v-\gamma| d_{q} t\right. \\
& +\left|{ }^{b} D_{q} f(a)\right| \int_{0}^{1} t|q t+\gamma v-1| d_{q} t+\left|{ }^{b} D_{q} f(b)\right| \int_{0}^{1}(1-t)|q t+\gamma v-1| d_{q} t \\
& \left.-\left|{ }^{b} D_{q} f(a)\right| \int_{0}^{v} t|q t+\gamma v-1| d_{q} t-\left|{ }^{b} D_{q} f(b)\right| \int_{0}^{v}(1-t)|q t+\gamma v-1| d_{q} t\right] \\
= & (b-a)\left[\left.\left(A_{1 q}(\gamma, v)+A_{2 q}(\gamma, v)-A_{3 q}(\gamma, v)\right)\right|^{b} D_{q} f(a) \mid\right. \\
& \left.+\left.\left(B_{1 q}(\gamma, v)+B_{2 q}(\gamma, v)-B_{3 q}(\gamma, v)\right)\right|^{b} D_{q} f(b) \mid\right],
\end{aligned}
$$

which completes the proof.

Remark 2 Under the assumptions of Theorem 4 with $q \rightarrow 1^{-}$, we have [25, Corollary 2.3].

Theorem 5 Suppose that $f:[a, b] \subset \mathbb{R} \rightarrow \mathbb{R}$ is $a q^{b}$-differentiable function on $(a, b)$ and ${ }^{b} D_{q} f$ is continuous and integrable on $[a, b]$. If $\left.\left.\right|^{b} D_{q} f\right|^{p_{1}}, p_{1}>1$, is convex on $[a, b]$, then we have following inequality:

$$
\begin{aligned}
& \left|\frac{1}{b-a} \int_{a}^{b} f(x)^{b} d_{q} x-\gamma[v f(a)+(1-v) f(b)]-(1-\gamma) f(v a+(1-v) b)\right| \\
& \leq(b-a)\left(C_{1 q}(\gamma, v)\right)^{1-\frac{1}{p_{1}}}\left(\left.\left.A_{2 q}(\gamma, v)\right|^{b} D_{q} f(a)\right|^{p_{1}}+\left.\left.B_{2 q}(\gamma, v)\right|^{b} D_{q} f(b)\right|^{p_{1}}\right)^{\frac{1}{p_{1}}} \\
& \quad+(b-a) v^{1-\frac{1}{p_{1}}}\left(\frac{v^{2}}{[2]_{q}}\left|{ }^{b} D_{q} f(a)\right|^{p_{1}}+\frac{v\left([2]_{q}-v\right)}{[2]_{q}}\left|{ }^{b} D_{q} f(b)\right|^{p_{1}}\right)^{\frac{1}{p_{1}}}
\end{aligned}
$$

where $0<q<1$.

Proof Taking the modulus in Lemma 1 and applying the well-known power-mean inequality for quantum integrals, we have

$$
\begin{aligned}
& \left|\frac{1}{b-a} \int_{a}^{b} f(x)^{b} d_{q} x-\gamma[v f(a)+(1-v) f(b)]-(1-\gamma) f(v a+(1-v) b)\right| \\
& =(b-a) \mid \int_{0}^{v}(q t+\gamma v-\gamma)^{b} D_{q} f(t a+(1-t) b) d_{q} t \\
& \quad+\int_{v}^{1}(q t+\gamma v-1)^{b} D_{q} f(t a+(1-t) b) d_{q} t \mid \\
& =(b-a) \mid \int_{0}^{1}(q t-\gamma v-1)^{b} D_{q} f(t a+(1-t) b) d_{q} t \\
& \quad+\int_{0}^{v}(1-\gamma)^{b} D_{q} f(t a+(1-t) b) d_{q} t \mid
\end{aligned}
$$




$$
\begin{aligned}
\leq & \left.(b-a) \int_{0}^{1}|q t-\gamma v-1|\right|^{b} D_{q} f(t a+(1-t) b) \mid d_{q} t \\
& +(b-a)(1-\gamma) \int_{0}^{v}\left|{ }^{b} D_{q} f(t a+(1-t) b)\right| d_{q} t \\
\leq & (b-a)\left(\int_{0}^{1}|q t+\gamma v-1| d_{q} t\right)^{1-\frac{1}{p_{1}}} \\
\quad & \left(\left.\left.\int_{0}^{1}|q t+\gamma v-1|\right|^{b} D_{q} f(t a+(1-t) b)\right|^{p_{1}} d_{q} t\right)^{\frac{1}{p_{1}}} \\
& +(b-a)(1-\gamma)\left(\int_{0}^{v} 1 d_{q} t\right)^{1-\frac{1}{p_{1}}}\left(\int_{0}^{v}\left|{ }^{b} D_{q} f(t a+(1-t) b)\right|^{p_{1}} d_{q} t\right)^{\frac{1}{p_{1}}} .
\end{aligned}
$$

By the convexity of $\left|{ }^{b} D_{q} f\right|^{p_{1}}$, we have

$$
\begin{aligned}
& \left|\frac{1}{b-a} \int_{a}^{b} f(x)^{b} d_{q} x-\gamma[v f(a)+(1-v) f(b)]-(1-\gamma) f(v a+(1-v) b)\right| \\
& \leq(b-a)\left(\int_{0}^{1}|q t+\gamma v-1| d_{q} t\right)^{1-\frac{1}{p_{1}}} \\
& \quad \times\left(\left|{ }^{b} D_{q} f(a)\right|^{p_{1}} \int_{0}^{1} t|q t+\gamma v-1| d_{q} t\right. \\
& \left.\quad+\left|{ }^{b} D_{q} f(b)\right|^{p_{1}} \int_{0}^{1}(1-t)|q t+\gamma v-1| d_{q} t\right)^{\frac{1}{p_{1}}} \\
& \quad+(b-a)(1-\gamma)\left(\int_{0}^{v} 1 d_{q} t\right){ }^{1-\frac{1}{p_{1}}} \\
& \quad \times\left(\int_{0}^{v}\left|{ }^{b} D_{q} f(a)\right|^{p_{1}} \int_{0}^{v} t d_{q} t+\left|{ }^{b} D_{q} f(b)\right|^{p_{1}} \int_{0}^{v}(1-t) d_{q} t\right)^{\frac{1}{p_{1}}} \\
& =(b-a)\left(C_{1 q}(\gamma, v)\right)^{1-\frac{1}{p_{1}}}\left(\left.\left.A_{2 q}(\gamma, v)\right|^{b} D_{q} f(a)\right|^{p_{1}}+\left.\left.B_{2 q}(\gamma, v)\right|^{b} D_{q} f(b)\right|^{p_{1}}\right)^{\frac{1}{p_{1}}} \\
& \quad+(b-a) v^{1-\frac{1}{p_{1}}}\left(\left.\left.\frac{v^{2}}{[2]_{q}}\right|^{b} D_{q} f(a)\right|^{p_{1}}+\frac{v\left([2]_{q}-v\right)}{[2]_{q}}\left|{ }^{b} D_{q} f(b)\right|^{p_{1}}\right)^{\frac{1}{p_{1}}},
\end{aligned}
$$

which completes the proof.

Remark 3 If we take $q \rightarrow 1^{-}$in Theorem 5 , then we have [25, Theorem 2.2].

Theorem 6 Let $f:[a, b] \subset \mathbb{R} \rightarrow \mathbb{R}$ be a $q^{b}$-differentiable function on $(a, b)$ and ${ }^{b} D_{q} f a$ continuous and integrable function on $[a, b]$. If $\left.\left.\right|^{b} D_{q} f\right|^{p_{1}}$ is convex on $[a, b]$ for some $p_{1}>1$ and $\frac{1}{r_{1}}+\frac{1}{p_{1}}=1$, then we have

$$
\begin{gathered}
\left|\frac{1}{b-a} \int_{a}^{b} f(x)^{b} d_{q} x-\gamma[v f(a)+(1-v) f(b)]-(1-\gamma) f(v a+(1-v) b)\right| \\
\leq(b-a)\left(u_{1 q}(\gamma, v)\right)^{\frac{1}{r_{1}}}\left(\frac{\left|{ }^{b} D_{q} f(a)\right|^{p_{1}}+\left.\left.q\right|^{b} D_{q} f(b)\right|^{p_{1}}}{[2]_{q}}\right)^{\frac{1}{p_{1}}} \\
\quad+(b-a) v^{\frac{1}{r_{1}}}\left(\frac{v^{2}}{[2]_{q}}\left|{ }^{b} D_{q} f(a)\right|^{p_{1}}+\left.\left.\frac{v\left([2]_{q}-v\right)}{[2]_{q}}\right|^{b} D_{q} f(b)\right|^{p_{1}}\right)^{\frac{1}{p_{1}}}
\end{gathered}
$$


where $0<q<1$ and

$$
u_{1 q}(\gamma, v)=\int_{0}^{1}|q t+\gamma v-1|^{r_{1}} d_{q} t .
$$

Proof Taking the modulus in the Lemma 1 and applying well-known Hölder's inequality for quantum integrals, we obtain

$$
\begin{aligned}
& \left|\frac{1}{b-a} \int_{a}^{b} f(x)^{b} d_{q} x-\gamma[v f(a)+(1-v) f(b)]-(1-\gamma) f(v a+(1-v) b)\right| \\
& =(b-a) \mid \int_{0}^{v}(q t+\gamma v-\gamma)^{b} D_{q} f(t a+(1-t) b) d_{q} t \\
& \quad+\int_{v}^{1}(q t+\gamma v-1)^{b} D_{q} f(t a+(1-t) b) d_{q} t \mid \\
& =(b-a) \mid \int_{0}^{1}(q t-\gamma v-1)^{b} D_{q} f(t a+(1-t) b) d_{q} t \\
& \quad+\int_{0}^{v}(1-\gamma)^{b} D_{q} f(t a+(1-t) b) d_{q} t \mid \\
& \leq\left.(b-a) \int_{0}^{1}|q t-\gamma v-1|\right|^{b} D_{q} f(t a+(1-t) b) \mid d_{q} t \\
& \quad+(b-a)(1-\gamma) \int_{0}^{v}\left|{ }^{b} D_{q} f(t a+(1-t) b)\right| d_{q} t \\
& \leq(b-a)\left(\int_{0}^{1}|q t+\gamma v-1|^{r_{1}} d_{q} t\right)^{\frac{1}{r_{1}}}\left(\int_{0}^{1}\left|{ }^{b} D_{q} f(t a+(1-t) b)\right|^{p_{1}} d_{q} t\right)^{\frac{1}{p_{1}}} \\
& \quad+(b-a)(1-\gamma)\left(\int_{0}^{v} 1^{r_{1}} d_{q} t\right)^{\frac{1}{r_{1}}}\left(\int_{0}^{v}\left|{ }^{b} D_{q} f(t a+(1-t) b)\right|^{p_{1}} d_{q} t\right)^{\frac{1}{p_{1}}} .
\end{aligned}
$$

Using the fact that $\left|{ }^{b} D_{q} f\right|^{p_{1}}$ is convex, we have

$$
\begin{aligned}
&\left|\frac{1}{b-a} \int_{a}^{b} f(x)^{b} d_{q} x-\gamma[v f(a)+(1-v) f(b)]-(1-\gamma) f(v a+(1-v) b)\right| \\
& \leq(b-a)\left(\int_{0}^{1}|q t+\gamma v-1|^{r_{1}} d_{q} t\right)^{\frac{1}{r_{1}}} \\
& \quad \times\left(\left|{ }^{b} D_{q} f(a)\right|^{p_{1}} \int_{0}^{1} t d_{q} t+\left|{ }^{b} D_{q} f(b)\right|^{p_{1}} \int_{0}^{1}(1-t) d_{q} t\right)^{\frac{1}{p_{1}}} \\
&\left.\quad+(b-a)(1-\gamma)\left(\int_{0}^{v} 1^{r_{1}} d_{q} t\right)\right)^{\frac{1}{r_{1}}} \\
& \quad \times\left(\left|{ }^{b} D_{q} f(a)\right|^{p_{1}} \int_{0}^{v} t d_{q} t+\left|{ }^{b} D_{q} f(b)\right|^{p_{1}} \int_{0}^{v}(1-t) d_{q} t\right)^{\frac{1}{p_{1}}} \\
&=(b-a)\left(u_{1 q}(\gamma, v)\right)^{\frac{1}{r_{1}}}\left(\frac{\left|{ }^{b} D_{q} f(a)\right|^{p_{1}}+\left.\left.q\right|^{b} D_{q} f(b)\right|^{p_{1}}}{[2]_{q}}\right)^{\frac{1}{p_{1}}} \\
& \quad+(b-a) v^{\frac{1}{r_{1}}}\left(\frac{v^{2}}{[2]_{q}}\left|{ }^{b} D_{q} f(a)\right|^{p_{1}}+\frac{v\left([2]_{q}-v\right)}{[2]_{q}}\left|{ }^{b} D_{q} f(b)\right|^{p_{1}}\right)^{\frac{1}{p_{1}}},
\end{aligned}
$$

which completes the proof. 
Remark 4 On taking limit as $q \rightarrow 1^{-}$in Theorem 6, we have [25, Theorem 2.8].

\section{Special cases}

In this section, we discus special cases of our main results and the relationship between the results presented herein and comparable results in the literature.

Remark 5 From Lemma 1, we have following observations:

(i) If we take $v=0$, then we obtain following new identity:

$$
\begin{gathered}
(b-a) \int_{0}^{1}(q t-1)^{b} D_{q} f(t a+(1-t) b) d_{q} t \\
=\frac{1}{b-a} \int_{a}^{b} f(x)^{b} d_{q} x-f(b) .
\end{gathered}
$$

(ii) By setting $v=1$ in Lemma 1, we obtain following new identity:

$$
\begin{gathered}
(b-a) \int_{0}^{1} q t^{b} D_{q} f(t a+(1-t) b) d_{q} t \\
=\frac{1}{b-a} \int_{a}^{b} f(x)^{b} d_{q} x-f(a) .
\end{gathered}
$$

(iii) Set $v=\frac{1}{[2]_{q}}$ to obtain the following new identity:

$$
\begin{aligned}
(b-a) & {\left[\int_{0}^{\frac{1}{[2]_{q}}}\left(q t-\frac{\gamma q}{[2]_{q}}\right)^{b} D_{q} f(t a+(1-t) b) d_{q} t\right.} \\
& \left.+\int_{\frac{1}{[2]_{q}}}^{1}\left(q t+\frac{\gamma}{[2]_{q}}-1\right)^{b} D_{q} f(t a+(1-t) b) d_{q} t\right] \\
= & \frac{1}{b-a} \int_{a}^{b} f(x)^{b} d_{q} x-\gamma \frac{f(a)+q f(b)}{[2]_{q}}-(1-\gamma) f\left(\frac{a+q b}{[2]_{q}}\right) .
\end{aligned}
$$

Remark 6 In the following, we give the different variants of Lemma 1.

(i) If we set $\gamma=0$, in Lemma 1 , we have following new identity:

$$
\begin{aligned}
& (b-a)\left[\int_{0}^{v} q t^{b} D_{q} f(t a+(1-t) b) d_{q} t+\int_{v}^{1}(q t-1)^{b} D_{q} f(t a+(1-t) b) d_{q} t\right] \\
& =\frac{1}{b-a} \int_{a}^{b} f(x)^{b} d_{q} x-f(v a+(1-v) b) .
\end{aligned}
$$

Specifically, for $v=\frac{1}{[2]_{q}}$, we have following midpoint-like identity given in [17, Lemma 2]:

$$
\begin{aligned}
& (b-a)\left[\int_{0}^{\frac{1}{[2]_{q}}} q t^{b} D_{q} f(t a+(1-t) b) d_{q} t+\int_{\frac{1}{[2]_{q}}}^{1}(q t-1)^{b} D_{q} f(t a+(1-t) b) d_{q} t\right] \\
& =\frac{1}{b-a} \int_{a}^{b} f(x)^{b} d_{q} x-f\left(\frac{a+q b}{[2]_{q}}\right) .
\end{aligned}
$$


(ii) Taking $\gamma=\frac{1}{3}$ in Lemma 1, we have following new identity:

$$
\begin{aligned}
(b-a) & {\left[\int_{0}^{v}\left(q t+\frac{1}{3} v-\frac{1}{3}\right)^{b} D_{q} f(t a+(1-t) b) d_{q} t\right.} \\
& \left.+\int_{v}^{1}\left(q t+\frac{1}{3} v-1\right)^{b} D_{q} f(t a+(1-t) b) d_{q} t\right] \\
= & \frac{1}{b-a} \int_{a}^{b} f(x)^{b} d_{q} x-\frac{1}{3}[v f(a)+(1-v) f(b)+2 f(v a+(1-v) b)] .
\end{aligned}
$$

Specifically, for $v=\frac{1}{[2]_{q}}$, we have Simpson-like integral identity given as follows:

$$
\begin{aligned}
(b-a) & {\left[\int_{0}^{\frac{1}{[2]_{q}}}\left(q t-\frac{q}{3\left([2]_{q}\right)}\right)^{b} D_{q} f(t a+(1-t) b) d_{q} t\right.} \\
& \left.+\int_{\frac{1}{[2]_{q}}}^{1}\left(q t+\frac{1}{3\left([2]_{q}\right)}-1\right)^{b} D_{q} f(t a+(1-t) b) d_{q} t\right] \\
= & \frac{1}{b-a} \int_{a}^{b} f(x)^{b} d_{q} x-\frac{1}{3}\left[\frac{f(a)+q f(b)}{[2]_{q}}+2 f\left(\frac{a+q b}{[2]_{q}}\right)\right] .
\end{aligned}
$$

(iii) If we set $\gamma=\frac{1}{2}$ in Lemma 1 , then we have following new identity:

$$
\begin{aligned}
(b-a) & {\left[\int_{0}^{v}\left(q t+\frac{1}{2} v-\frac{1}{2}\right)^{b} D_{q} f(t a+(1-t) b) d_{q} t\right.} \\
& \left.+\int_{v}^{1}\left(q t+\frac{1}{2} v-1\right)^{b} D_{q} f(t a+(1-t) b) d_{q} t\right] \\
= & \frac{1}{b-a} \int_{a}^{b} f(x)^{b} d_{q} x-\frac{1}{2}[v f(a)+(1-v) f(b)+f(v a+(1-v) b)] .
\end{aligned}
$$

Specifically, for $v=\frac{1}{[2]_{q}}$, we get following averaged midpoint-trapezoid-type integral identity:

$$
\begin{aligned}
(b-a) & {\left[\int_{0}^{\frac{1}{[2]_{q}}}\left(q t-\frac{q}{2\left([2]_{q}\right)}\right)^{b} D_{q} f(t a+(1-t) b) d_{q} t\right.} \\
& \left.+\int_{\frac{1}{[2]_{q}}}^{1}\left(q t+\frac{1}{2\left([2]_{q}\right)}-1\right)^{b} D_{q} f(t a+(1-t) b) d_{q} t\right] \\
= & \frac{1}{b-a} \int_{a}^{b} f(x)^{b} d_{q} x-\frac{1}{2}\left[\frac{f(a)+q f(b)}{[2]_{q}}+f\left(\frac{a+q b}{[2]_{q}}\right)\right] .
\end{aligned}
$$

(iv) By setting $\gamma=1$ in Lemma 1,we have following new identity

$$
\begin{array}{r}
(b-a)\left[\int_{0}^{v}(q t+v-1)^{b} D_{q} f(t a+(1-t) b) d_{q} t\right. \\
\left.+\int_{v}^{1}(q t+v-1)^{b} D_{q} f(t a+(1-t) b) d_{q} t\right] \\
=\frac{1}{b-a} \int_{a}^{b} f(x)^{b} d_{q} x-[v f(a)+(1-v) f(b)] .
\end{array}
$$


Specifically, for $v=\frac{1}{[2]_{q}}$, we have following trapezoid-like integral identity given in [17, Lemma 1]:

$$
\begin{aligned}
(b-a) & {\left[\int_{0}^{\frac{1}{[2]_{q}}}\left(q t+\frac{1}{[2]_{q}}-1\right)^{b} D_{q} f(t a+(1-t) b) d_{q} t\right.} \\
+ & \left.\int_{\frac{1}{[2]_{q}}}^{1}\left(q t+\frac{1}{[2]_{q}}-1\right)^{b} D_{q} f(t a+(1-t) b) d_{q} t\right] \\
= & \frac{1}{b-a} \int_{a}^{b} f(x)^{b} d_{q} x-\frac{f(a)+q f(b)}{[2]_{q}} .
\end{aligned}
$$

Corollary 2 Under the assumptions of Theorem 4 with $v=\frac{1}{[2]_{q}}$, we have following new inequality:

$$
\begin{aligned}
& \left|\frac{1}{b-a} \int_{a}^{b} f(x)^{b} d_{q} x-\gamma\left[\frac{f(a)+q f(b)}{[2]_{q}}\right]-(1-\gamma) f\left(\frac{a+q b}{[2]_{q}}\right)\right| \\
& \leq(b-a)\left[\left(A_{1 q}\left(\gamma, \frac{1}{[2]_{q}}\right)+A_{2 q}\left(\gamma, \frac{1}{[2]_{q}}\right)-A_{3 q}\left(\gamma, \frac{1}{[2]_{q}}\right)\right)\left|{ }^{b} D_{q} f(a)\right|\right. \\
& \left.\quad+\left(B_{1 q}\left(\gamma, \frac{1}{[2]_{q}}\right)+B_{2 q}\left(\gamma, \frac{1}{[2]_{q}}\right)-B_{3 q}\left(\gamma, \frac{1}{[2]_{q}}\right)\right)\left|{ }^{b} D_{q} f(b)\right|\right] .
\end{aligned}
$$

Remark 7 We now deduce some more inequalities from Corollary 2.

(i) By putting $\gamma=0$ in Corollary 2, we obtain following midpoint-type inequality [17,

Theorem 5]:

$$
\begin{aligned}
& \left|\frac{1}{b-a} \int_{a}^{b} f(x)^{b} d_{q} x-f\left(\frac{a+q b}{[2]_{q}}\right)\right| \\
& \quad \leq(b-a)\left[\left|{ }^{b} D_{q} f(a)\right| \frac{3 q}{\left([2]_{q}\right)^{3}\left([3]_{q}\right)}+\left|{ }^{b} D_{q} f(b)\right| \frac{-q+2 q^{2}+q^{3}}{\left([2]_{q}\right)^{3}\left([3]_{q}\right)}\right] .
\end{aligned}
$$

(ii) If we take $\gamma=\frac{1}{3}$ in Corollary 2, then we get following Simpson's-type inequality:

$$
\begin{aligned}
& \left|\frac{1}{b-a} \int_{a}^{b} f(x)^{b} d_{q} x-\frac{1}{3}\left[\frac{f(a)+q f(b)}{[2]_{q}}+2 f\left(\frac{a+q b}{[2]_{q}}\right)\right]\right| \\
& \leq(b-a)\left[\left(A_{1 q}\left(\frac{1}{3}, \frac{1}{[2]_{q}}\right)+A_{2 q}\left(\frac{1}{3}, \frac{1}{[2]_{q}}\right)-A_{3 q}\left(\frac{1}{3}, \frac{1}{[2]_{q}}\right)\right)\left|{ }^{b} D_{q} f(a)\right|\right. \\
& \left.\quad+\left(B_{1 q}\left(\frac{1}{3}, \frac{1}{[2]_{q}}\right)+B_{2 q}\left(\frac{1}{3}, \frac{1}{[2]_{q}}\right)-B_{3 q}\left(\frac{1}{3}, \frac{1}{[2]_{q}}\right)\right)\left|{ }^{b} D_{q} f(b)\right|\right] .
\end{aligned}
$$

Specifically for $q \rightarrow 1^{-}$, we have following inequality given by Alomori et al. [9, Corollary 1]:

$$
\left|\frac{1}{b-a} \int_{a}^{b} f(x) d x-\frac{1}{3}\left[\frac{f(a)+f(b)}{2}+2 f\left(\frac{a+b}{2}\right)\right]\right| \leq \frac{5(b-a)}{72}\left[\left|f^{\prime}(b)\right|+\left|f^{\prime}(a)\right|\right] .
$$


(iii) Take $\gamma=\frac{1}{2}$ in Corollary 2 to obtain the following averaged midpoint-trapezoidtype inequality:

$$
\begin{aligned}
& \left|\frac{1}{b-a} \int_{a}^{b} f(x)^{b} d_{q} x-\frac{1}{2}\left[\frac{f(a)+q f(b)}{[2]_{q}}+f\left(\frac{a+q b}{[2]_{q}}\right)\right]\right| \\
& \leq(b-a)\left[\left(A_{1 q}\left(\frac{1}{2}, \frac{1}{[2]_{q}}\right)+A_{2 q}\left(\frac{1}{2}, \frac{1}{[2]_{q}}\right)-A_{3 q}\left(\frac{1}{2}, \frac{1}{[2]_{q}}\right)\right)\left|{ }^{b} D_{q} f(a)\right|\right. \\
& \left.\quad+\left(B_{1 q}\left(\frac{1}{2}, \frac{1}{[2]_{q}}\right)+B_{2 q}\left(\frac{1}{2}, \frac{1}{[2]_{q}}\right)-B_{3 q}\left(\frac{1}{2}, \frac{1}{[2]_{q}}\right)\right)\left|{ }^{b} D_{q} f(b)\right|\right] .
\end{aligned}
$$

Specifically, for $q \rightarrow 1^{-}$, we obtain the following inequality in [44, Corollary 3.4]:

$$
\begin{aligned}
& \left|\frac{1}{b-a} \int_{a}^{b} f(x) d x-\frac{1}{2}\left[\frac{f(a)+f(b)}{2}+f\left(\frac{a+b}{2}\right)\right]\right| \\
& \quad \leq \frac{b-a}{16}\left[\left|f^{\prime}(b)\right|+\left|f^{\prime}(a)\right|\right] .
\end{aligned}
$$

(iv) If we take $\gamma=1$ in Corollary 2, then we obtain following trapezoid-type integral inequality:

$$
\begin{aligned}
& \left|\frac{1}{b-a} \int_{a}^{b} f(x)^{b} d_{q} x-\frac{f(a)+q f(b)}{[2]_{q}}\right| \\
& \quad \leq(b-a)\left[\left|{ }^{b} D_{q} f(a)\right| \frac{q^{2}\left(1+4 q+q^{2}\right)}{\left([2]_{q}\right)^{4}\left([3]_{q}\right)}+\left|{ }^{b} D_{q} f(b)\right| \frac{q^{2}\left(1+3 q^{2}+2 q^{3}\right)}{\left([2]_{q}\right)^{4}\left([3]_{q}\right)}\right]
\end{aligned}
$$

which is given by Budak in [17, Theorem 3].

Remark 8 Under the assumptions of Theorems 5 and 6 with $v=\frac{1}{[2]_{q}}$, we may choose $\gamma=$ $0, \gamma=\frac{1}{3}, \gamma=\frac{1}{2}$, and $\gamma=1$, to obtain the midpoint-like integral inequality, the Simpsonlike integral inequality, the averaged midpoint-trapezoid-like integral inequality and the trapezoid-like integral inequality, respectively.

\section{Conclusions}

In this paper, some new bounds of Hermite-Hadamard inequalities for convex functions by applying the notions of the newly defined $q^{b}$-integral and $q^{b}$-derivative are obtained. It is shown that the results proved herein are generalizations of the existing comparable results in the literature, like the results proved in $[9,17,25,44]$. It is an interesting and new problem that the upcoming researchers can offer similar inequalities for different kinds of convexity in their future work.

\section{Acknowledgements}

We are thankful to the editor and referees for their careful reading and valuable suggestions which have made the article reader friendly.

\section{Funding}

The work was supported by the Natural Science Foundation of China (Grant Nos. 61673169, 11301127, 11701176, 11626101, 11601485, 11971241). 


\section{Competing interests}

The authors declare that they have no competing interests.

\section{Authors' contributions}

The study was carried out in collaboration of all authors. All authors read and approved the final manuscript.

\section{Author details}

${ }^{1}$ College of Mathematics and Finance, Xiangnan University, Chenzhou 423000, P.R. China. ${ }^{2}$ Jiangsu Key Laboratory for NSLSCS, School of Mathematical Sciences, Nanjing Normal University, Nanjing, China. ${ }^{3}$ Department of Mathematics, Faculty of Science and Arts, Düzce University, Düzce, Turkey. ${ }^{4}$ Department of Mathematics, Government College University, Lahore 54000, Pakistan. ${ }^{5}$ Department of Mathematics, Huzhou University, Huzhou 313000, China.

\section{Publisher's Note}

Springer Nature remains neutral with regard to jurisdictional claims in published maps and institutional affiliations.

Received: 15 October 2020 Accepted: 18 April 2021 Published online: 29 April 2021

\section{References}

1. Adil Khan, M., Mohammad, N., Nwaeze, E.R., Chu, Y.-M.: Quantum Hermite-Hadamard inequality by means of a Green function. Adv. Differ. Equ. 2020(1), 99 (2020)

2. Ali, M.A., Abbas, M., Budak, H., Agarwal, P., Murtaza, G., Chu, Y.-M.: New quantum boundaries for quantum Simpson's and quantum Newton's type inequalities for preinvex functions. Adv. Differ. Equ. 2021, 64 (2021). https://doi.org/10.1186/s13662-021-03226-X

3. Ali, M.A., Alp, N., Budak, H., Chu, Y.-M., Zhang, Z.: On some new quantum midpoint type inequalities for twice quantum differentiable convex functions. Open Math. (2021, in press)

4. Ali, M.A., Budak, H., Abbas, M., Chu, Y.-M.: Quantum Hermite-Hadamard-type inequalities for functions with convex absolute values of second $q^{b}$-derivatives. Adv. Differ. Equ. 2021, 7 (2021). https://doi.org/10.1186/s13662-020-03163-1

5. Ali, M.A., Budak, H., Akkurt, A., Chu, Y.-M.: Quantum Ostrowski type inequalities for twice quantum differentiable functions in quantum calculus. Open Math. (2021, in press)

6. Ali, M.A., Budak, H., Zhang, Z., Yildrim, H.: Some new Simpson's type inequalities for co-ordinated convex functions in quantum calculus. Math. Methods Appl. Sci. https://doi.org/10.1002/mma.7048

7. Ali, M.A., Chu, Y.-M., Budak, H., Akkurt, A., Yıldırım, H., Zahid, M.A.: Quantum variant of Montgomery identity and Ostrowski-type inequalities for the mappings of two variables. Adv. Differ. Equ. 2021, 25 (2021). https://doi.org/10.1186/s13662-020-03195-7

8. Almeida, R., Torres, D.F.M.: Non-differentiable variational principles in terms of a quantum operator. Math. Methods Appl. Sci. 34, 2231-2241 (2011)

9. Alomari, M., Darus, M., Dragomir, S.S.: New inequalities of Simpson's type for s-convex functions with applications Res. Rep. Collect. 12(4) (2009)

10. Alp, N., Sarikaya, M.Z.: Hermite-Hadamard's type inequalities for coordinated convex functions on quantum integral. Submitted. https://www.researchgate.net/publication/329814922

11. Alp, N., Sarikaya, M.Z., Kunt, M., Iscan, I.: q-Hermite-Hadamard inequalities and quantum estimates for midpoint type inequalities via convex and quasi-convex functions. J. King Saud Univ., Sci. 30, 193-203 (2018)

12. Anastassiou, G.A.: Intelligent Mathematics: Computational Analysis. Springer, New York (2011)

13. Askey, R., Wilson, J.: Some basic hypergeometric orthogonal polynomials that generalize the Jacobi polynomials. Mem. Am. Math. Soc. 54, 1-55 (1985)

14. B.-Mohsin, B., Awan, M.U., Noor, M.A., Riahi, L., Noor, K.I., Almutairi, B.: New quantum Hermite-Hadamard inequalities utilizing harmonic convexity of the functions. IEEE Access 7, 20479-20483 (2019)

15. Bermudo, S., Kórus, P., Valdés, J.N.: On q-Hermite-Hadamard inequalities for general convex functions. Acta Math. Hung. 162, 364-374 (2020)

16. Brito, A.M.C., Cruz, D.A.: Symmetric Quantum Calculus. PhD Thesis, Aveiro University, Aveiro, Portugal (2012)

17. Budak, H.: Some trapezoid and midpoint type inequalities for newly defined quantum integrals. Proyecciones (in press)

18. Budak, H., Ali, M.A., Tarhanaci, M.: Some new quantum Hermite-Hadamard-like inequalities for coordinated convex functions. J. Optim. Theory Appl. 186, 899-910 (2020)

19. Budak, H., Ali, M.A., Tunc, T.: Quantum Ostrowski-type integral inequalities for functions of two variables. Math. Methods Appl. Sci. (2021). https://doi.org/10.1002/mma.7153

20. Budak, H., Erden, S., Ali, M.A.: Simpson and Newton type inequalities for convex functions via newly defined quantum integrals. Math. Methods Appl. Sci. (2020)

21. Cresson, J., Frederico, G.S.F., Torres, D.F.M.: Constants of motion for non-differentiable quantum variational problems. Topol. Methods Nonlinear Anal. 33, 217-231 (2009)

22. Ernst, T.: The History of Q-Calculus and New Method. Department of Mathematics, Uppsala University, Sweden (2000)

23. Ernst, T:: A Comprehensive Treatment of $q$-Calculus. Springer, Basel (2012)

24. Gauchman, H.: Integral inequalities in q-calculus. Comput. Math. Appl. 47, 281-300 (2004)

25. Işcan, I.: A new generalization of some integral inequalities and their applications. Int. J. Eng. Appl. Sci. 3(3), 17-27 (2013)

26. Ismail, M.E.H., Simeonov, P.: q-Difference operators for orthogonal polynomials. J. Comput. Appl. Math. 233, 749-761 (2009)

27. Jackson, F.H.: On a q-definite integrals. Q. J. Pure Appl. Math. 41, 193-203 (1910)

28. Kac, V., Cheung, P.: Quantum Calculus. Springer, Berlin (2001) 
29. Li, Y.-X., Muhammad, T., Bilal, M., Altaf Khan, M., Ahmadian, A., Pansera, B.A.: Fractional simulation for Darcy-Forchheimer hybrid nanoliquid flow with partial slip over a spinning disk. Alex. Eng. J. 60, 4787-4796 (2021)

30. Liu, W., Hefeng, Z.: Some quantum estimates of Hermite-Hadamard inequalities for convex functions. J. Appl. Anal. Comput. 7(2), 501-522 (2017)

31. Noor, M., Noor, K., Awan, M.: Quantum Ostrowski inequalities for q-differentiable convex functions. J. Math. Inequal. 10(4), 1013-1018 (2016)

32. Noor, M.A., Critescu, G., Awan, M.U.: Bounds having Riemann type quantum integrals via strongly convex functions. Studia Sci. Math. Hung. 54(2), 221-240 (2017)

33. Noor, M.A., Noor, K.I., Awan, M.U.: Some quantum estimates for Hermite-Hadamard inequalities. Appl. Math. Comput. 251, 675-679 (2015)

34. Noor, M.A., Noor, K.I., Awan, M.U.: Some quantum integral inequalities via preinvex functions. Appl. Math. Comput. 269, 242-251 (2015)

35. Nwaeze, E.R., Tameru, A.M.: New parameterized quantum integral inequalities via $\eta$-quasiconvexity. Adv. Differ. Equ. 2019(1), $425(2019)$

36. Page, D.N.: Information in black hole radiation. Phys. Rev. Lett. 71, 3743-3746 (1993)

37. Sudsutad, W., Ntouyas, S.K., Tariboon, J.: Quantum integral inequalities for convex functions. J. Math. Inequal. 9(3), 781-793 (2015)

38. Tariboon, J., Ntouyas, S.K.: Quantum calculus on finite intervals and applications to impulsive difference equations. Adv. Differ. Equ. 282, 1-19 (2013)

39. Vivas-Cortez, M., Ali, M.A., Kashuri, A., Sial, I.B., Zhang, Z.: Some new Newton's type integral inequalities for co-ordinated convex functions in quantum calculus. Symmetry 12(9), 1476 (2020)

40. Vivas-Cortez, M., Kashuri, A., Liko, R., Hernández Hernández, J.E.: Quantum trapezium-type inequalities using generalized f-convex functions. Axioms 9(1), 1-14 (2020)

41. Vivas-Cortez, M., Liko, R., Kashuri, A., Hernández Hernández, J.E.: New quantum estimates of trapezium-type inequalities for generalized $\phi$-convex functions. Mathematics 7(11), 1-19 (2019)

42. Vivas-Cortez, M., Liko, R., Kashuri, A., Hernández Hernández, J.E.: Quantum estimates of Ostrowski inequalities for generalized $\phi$-convex functions. Symmetry 11(12), 1-16 (2019)

43. Vivas-Cortez, M., Liko, R., Kashuri, A., Hernández Hernández, J.E.: Some new q-integral inequalities using generalized quantum Montgomery identity via preinvex functions. Symmetry 12(4), 1-15 (2020)

44. Xi, B.Y., Qi, F.: Some Hermite-Hadamard type inequalities for differentiable convex functions and applications. Hacet. J. Math. Stat. 42(3), 243-257 (2013)

45. Youm, D.: q-deformed conformal quantum mechanics. Phys. Rev. D 62, 095009 (2000)

46. Zhang, Y., Du, T.S., Wang, H., Shen, Y.J.: Different types of quantum integral inequalities via $(\alpha, m)$-convexity. J. Inequal. Appl. 2018(1), 264 (2018)

47. Zhuang, H., Liu, W., Park, J.: Some quantum estimates of Hermite-Hadmard inequalities for quasi-convex functions. Mathematics 7, 152 (2019)

\section{Submit your manuscript to a SpringerOpen ${ }^{\circ}$ journal and benefit from:}

- Convenient online submission

- Rigorous peer review

- Open access: articles freely available online

- High visibility within the field

- Retaining the copyright to your article

Submit your next manuscript at $\boldsymbol{s p r i n g e r o p e n . c o m ~}$ 